\title{
Oridonin induces apoptosis in HGC-27 cells by activating the JNK signaling pathway
}

\author{
DONG-LIN REN $^{1 *}$, ROSHAN ARA GHOORUN ${ }^{1 *}$, XIAO-HUA WU ${ }^{2}$, \\ HONG-LEI CHEN $^{3}$, QIAN ZHOU ${ }^{1}$ and XIAO-BIN WU ${ }^{4}$ \\ ${ }^{1}$ Department of Colorectal Surgery, The Sixth Affiliated Hospital of Sun Yat-sen University, Guangzhou, Guangdong 510655;
${ }^{2}$ Department of Oncology, Longhu People's Hospital, Shantou, Guangdong 515000; Departments of ${ }^{3}$ Endoscopy and
${ }^{4}$ Gastrointestinal Surgery, The Eighth Affiliated Hospital of Sun Yat-sen University, Shenzhen, Guangdong 518033, P.R. China
}

Received May 21, 2019; Accepted October 10, 2019

DOI: $10.3892 / \mathrm{ol} .2019 .11104$

\begin{abstract}
Gastric cancer (GC) is a very common type of cancer. Although current treatment modalities include surgical resection and chemotherapy, many patients are either not eligible for radical resection or have a poor response to chemotherapy. Due to the complex features of the disease, there is a need for complementary therapy. In the present study, the effects of oridonin on cell proliferation, invasion and apoptosis were assessed in the HGC-27 cell line using the Cell Counting Kit-8 assay, real-time cell analysis, and an Annexin V-FITC/propidium iodide (PI) detection kit, respectively. The effect of oridonin on apoptosis, through the JNK pathway, was also investigated using western blotting. The present study demonstrated that oridonin can suppress cell viability and inhibit cell proliferation by inducing $\mathrm{G}_{2} / \mathrm{M}$ arrest. Oridonin also induced caspase-dependent apoptosis in cells by activating the phosphorylated-JNK/C-JUN pathway. These results demonstrate the potential of oridonin as a potential therapeutic compound for the treatment of GC.
\end{abstract}

\section{Introduction}

Gastric cancer (GC) is one of the most common cancer types worldwide. In 2018, 26,240 new cases were identified in the United States of America (1). Although current treatment modalities include surgical resection and chemotherapy, many patients are not eligible for radical resection or have a poor

Correspondence to: $\mathrm{Dr}$ Xiao-Bin $\mathrm{Wu}$, Department of Gastrointestinal Surgery, The Eighth Affiliated Hospital of Sun Yat-sen University, 3025 Shennan Middle Road, Shenzhen, Guangdong 518033, P.R. China

mail: xiaobinwoo@hotmail.com

*Contributed equally

Key words: oridonin, gastric cancer, apoptosis, caspase, JNK pathway response to chemotherapy (2). Overall, the 5-year survival rate for GC patients is at an unacceptably low rate of $31 \%$ (1).

Natural products are one of the key foundations of medical history. Due to the complex features of GC, there is a need for complementary therapy such as herbal medication. Molecular properties such as high chemical heterogeneity and biocompatible features make herbal therapy an optimal scaffold for drug discovery. More than $50 \%$ of commonly used drugs in clinical practice are derived from natural products (3). In oncology, this rate increases to $>60 \%$ (4). Oridonin has been shown to induce apoptosis and autophagy in several cancer cell lines (5-7), including GC cell lines (8). Nevertheless, the molecular mechanisms of how oridonin contributes to $\mathrm{GC}$ treatment remain poorly understood.

Oridonin has been found to activate mitogen-activated kinases (MAPKs) (9), which play important roles in cell proliferation and apoptosis (10). However, the exact MAPK pathway through which oridonin induces apoptosis in GC remains elusive. Herein, the underlying mechanisms through which oridonin contributes to apoptosis was investigated. The HGC-27 cell line was used for this study, as the efficacy of oridonin has already been reported (8). The effect of oridonin on proliferation and apoptosis was assessed and the possible MAPK pathway responsible for activating the observed caspase-dependent apoptosis was investigated.

\section{Materials and methods}

Cell culture. The HGC-27 cell line, obtained from The Cell Bank of Type Culture Collection of the Chinese Academy of Sciences was used for this investigation. The cells were cultured with RPMI-1640 supplemented with $10 \%$ fetal bovine serum, $100 \mathrm{U} / \mathrm{ml}$ penicillin and $10 \mu \mathrm{g} / \mathrm{ml}$ streptomycin (Gibco; Thermo Fisher Scientific, Inc.) in a humidified atmosphere at $37^{\circ} \mathrm{C}$ and $5 \% \mathrm{CO}_{2}$ atmosphere. SP600125 (Sigma-Aldrich; Merck KGaA) was used as a general inhibitor of JNK. Cells $\left(3 \times 10^{3}\right.$ per well) were treated with $5 \mu \mathrm{M} \mathrm{SP} 600125$ at $37^{\circ} \mathrm{C}$ for $24 \mathrm{~h}$.

Cell Counting Kit-8 assay. The CCK-8 assay was utilized to investigate the cytotoxic effect of oridonin on HGC-27 cells. Cells $\left(3 \times 10^{3}\right.$ per well) were seeded onto 96 -well plates. 
Different concentrations of oridonin $(2.5,5,7.5,10$ and $15 \mu \mathrm{M})$ were added to the cells. Following an incubation period of 12 , 24,48 and $72 \mathrm{~h}$, the cells were treated with $20 \mu \mathrm{l}$ of CCK- 8 solution. These four time points were selected to assess the change in the overall survival of HGC-27 cells under different time periods. The optical absorbance was assessed at $450 \mathrm{~nm}$ utilizing a 96-well plate reader (Thermo Fisher Scientific, Inc.). All experiments were performed three times.

Colony formation. Various concentrations of oridonin were allocated to the cells, after which cells were incubated for 7 days. For assay termination, the cells were irrigated and stabilized in ice-cold $100 \%$ methanol for $5 \mathrm{~min}$, followed by staining with crystal violet dye (Beyotime Institute of Biotechnology) for $30 \mathrm{~min}$. Images of colonies were captured using an Epson scanner (Suwa).

Real-time cell impedance. The rate of cell proliferation in a real-time cell analysis (RTCA) was performed at 24, 48, 72 and $96 \mathrm{~h}$, as per the conventional instructions given by Roche Applied Science. In brief, $100 \mu 1$ of the mixture was added to every plate, which was already filled with $2 \times 10^{3}$ cells. Cell impedance was assessed with the xCELLigence RTCA DP instrument (ACEA Biosciences).

Cell cycle analysis. The GC cells were first treated with oridonin. Thereafter, the cells were settled with $66 \%$ ethanol and kept at $4{ }^{\circ} \mathrm{C}$ overnight. The following day, the ethanol was removed, irrigated and the cells were dyed with PBS and propidium iodide (PI) respectively. Cell cycle distribution was subsequently assessed utilizing FASCanto II flow cytometry (BD Biosciences), according to the manufacturer's protocols.

Cell apoptosis analysis. The Annexin V-FITC/propidium iodide (PI) detection kit (Nanjing KeyGen Biotech Co., Ltd.) was used to monitor cell apoptosis. Cells were first treated in the presence or absence of oridonin for the allocated period. Subsequently, the cells were detached and resuspended in $500 \mu \mathrm{l}$ binding buffer containing $5 \mu \mathrm{l}$ of PI and $5 \mu \mathrm{l}$ of Annexin V-FITC. Thereafter, the cells were incubated in the dark at $25^{\circ} \mathrm{C}$ for $15 \mathrm{~min}$ prior to analysis. Cell apoptosis was analyzed at $24 \mathrm{~h}$.

Western blotting. Proteins were extracted from the cells using RIPA buffer (cat. no. 89901; Thermo Fisher Scientific, Inc.) at $4^{\circ} \mathrm{C}$. Protein concentration was measured using the BCA protein assay kit (Thermo Fisher Scientific, Inc.) according to the manufacturer's protocols. Proteins ( $30 \mu \mathrm{g} / \mathrm{lane})$ were separated by $10 \%$ SDS-PAGE and transferred onto PVDF membranes. The membranes were subsequently incubated overnight at $4{ }^{\circ} \mathrm{C}$ with $5 \%$ nonfat dry milk and the primary antibodies against PARP (cat. no. 9532; from Cell Signaling Technology, Inc.; 1:1,000), pro-caspase-3 (cat. no. 9662, 1:1,000), cleaved-caspase-3 (cat. no. 9502, 1:1,000), pro-caspase-8 (cat. no. 9746, 1:1,000), Mcl-1 (cat. no. 94296, 1:1,000), Bcl-xl (cat. no. 2764, 1:1,000), Bcl-2 (cat. no. 15071, 1:1,000), Xiap (cat. no. 15071, 1:1,000), survivin (cat. no. 2808, 1:1,000), cyclin B1 (cat. no. 12231, 1:1,000), p53 (cat. no. 48818, 1:1,000), p21 (cat. no. 2947, 1:1,000; all from Cell Signaling Technology, Inc.), cleaved-caspase-9 (cat. no. ab2324; Abcam; 1:1,000), pro-caspase-9 (cat. no. ab2013; Abcam; 1:1,000) and $\beta$-actin (cat. no. 3700, 1:10,000; Cell Signaling Technology, Inc.). The following day, the membranes were washed with PBST and further incubated for $2 \mathrm{~h}$ at $4^{\circ} \mathrm{C}$ with anti-mouse $\operatorname{IgG}$ (cat. no. 16402-1-AP; ProteinTech Group, Inc.; 1:1,500) and anti-rabbit IgG (cat. no. 13688-1-AP, ProteinTech Group, Inc.; 1:1,500) horseradish peroxidase conjugated secondary antibodies. Next, the membranes were bands were detected using enhanced chemiluminescence substrate (Generay Biotech Co., Ltd.).

Statistical analysis. Results in this study represent three independent experiments. All data are expressed as means \pm standard deviation. ANOVA followed by Tukey's multiple comparison tests were performed utilizing GraphPad Prism (GraphPad Software, Inc.). $\mathrm{P}<0.05$ were considered to indicate a statistically significant difference.

\section{Results}

Cytotoxic effects of oridonin on HGC-27 cells. The CCK-8 assay was first used to determine whether oridonin could effectively decrease the viability of HGC-27 cells. Effectively, oridonin increased the cytotoxicity of GC cells in an evident dose- and time-dependent manner in the HGC-27 line. Following the treatment of cells with oridonin for $72 \mathrm{~h}$, the IC50 values increased from 3.61 to $21.11 \mu \mathrm{M}$.

In order to assess the dose-response effect of oridonin, the HGC-27 cells were cultured in 2.5, 5, 10, 15, 25, 50, 75 and $100 \mu \mathrm{M}$ oridonin for $48 \mathrm{~h}$ to assess the cytotoxic effects of the drug (Fig. 1A). Oridonin decreased HGC-27 cell viability in a dose-dependent manner when used at concentrations of between 2.5 and $25 \mu \mathrm{M}$ (Fig. 1B).

Oridonin induces proliferation in HGC-27 cells. In order to investigate the effect of oridonin on proliferation, $\mathrm{HGC}-27$ cells were exposed to different doses of oridonin $(2.5-10 \mu \mathrm{M})$ for 7 days. The cells exposed to oridonin formed fewer colonies (Fig. 2A) in comparison with the control groups. These results further demonstrate the dose-dependent inhibitory effect of oridonin on HGC-27 cell proliferation (Fig. 2B).

Oridonin induces apoptotic cell death in $\mathrm{HGC}-27$ cells. In order to assess the properties of oridonin on cell death in HGC-27 cells, flow cytometric analysis was conducted. The apoptotic cell ratio was 15.7 in the control group. Upon adding 10,15 and $20 \mu \mathrm{M}$ oridonin to the cells, an increase in apoptotic cells was observed at $24 \mathrm{~h}(26.3,50.1$ and 52.4, respectively; $\mathrm{P}<0.05$; Fig. 2C). Since apoptosis was observed at $24 \mathrm{~h}$, further time points were not investigated. Thus, oridonin induces cell death in the HGC-27 cells via apoptosis in a dose-dependent manner.

Oridonin arrests cell cycle progression of GC cells. It was hypothesized that cell cycle arrest was the underlying mechanism of oridonin-induced inhibition of cell proliferation, which was investigated using flow cytometry. Following exposure to 15 and $20 \mu \mathrm{M}$ oridonin for $12 \mathrm{~h}$, an increased population of cells were observed at the $\mathrm{G}_{2} / \mathrm{M}$ phase (Fig. 2D). The findings of the present study suggest that oridonin induces cell death 

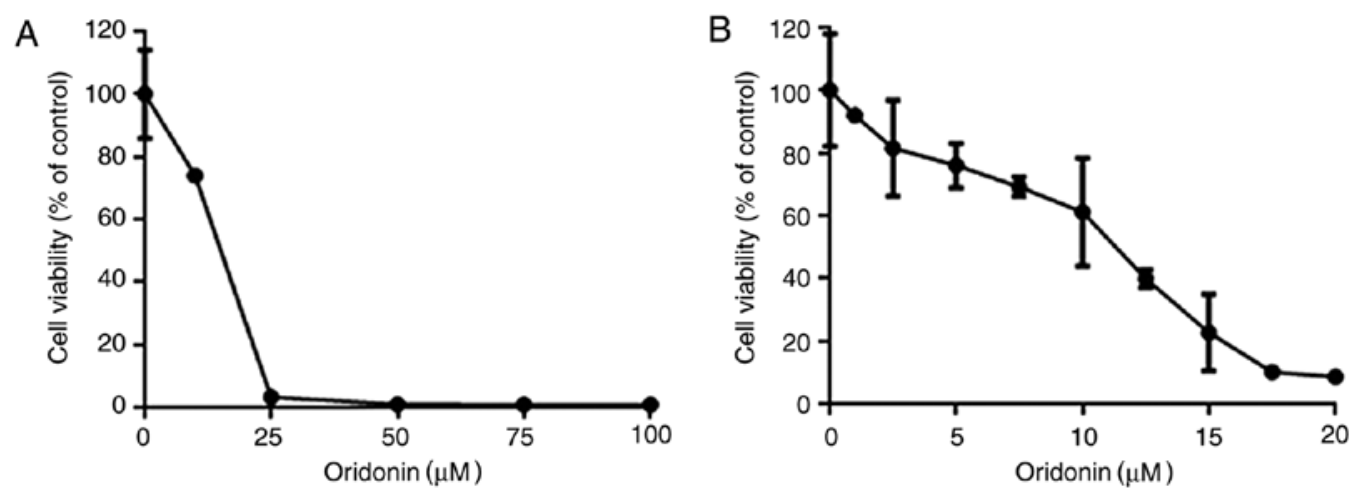

Figure 1. Viability of HGC-27 cells is inhibited by oridonin. (A) Oridonin increases the cytotoxicity of HGC-27 cells in a dose-dependent manner. (B) Decrease in cell death is observed at lower concentrations of oridonin.

A
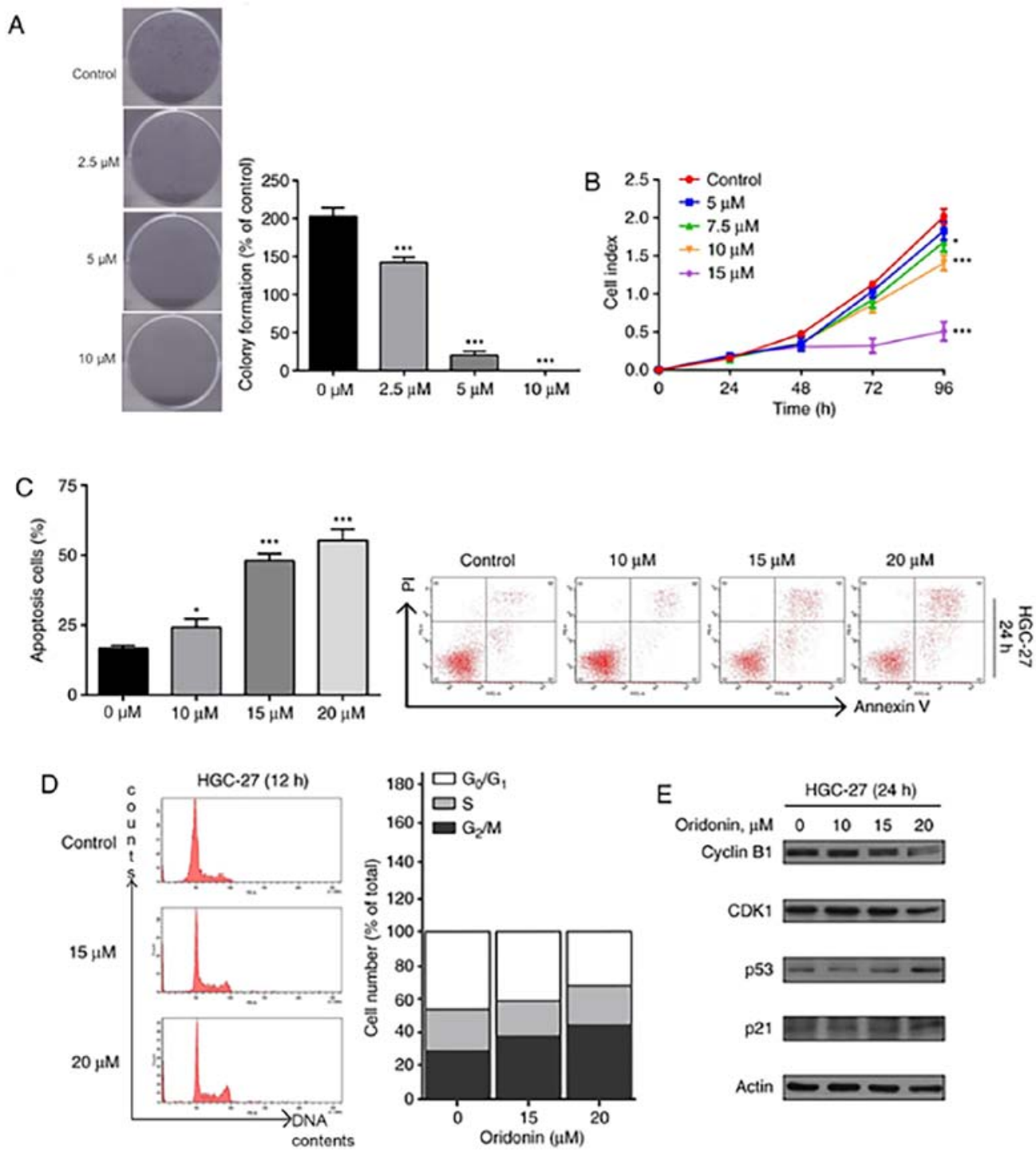

Figure 2. Oridonin induces inhibition of cell proliferation. (A) Colony-formation assay of HGC-27 cells, following treatment with oridonin for 7 days. (B) The real-time cell impedance assay results demonstrate the rate of HGC-27 cell proliferation. (C) Apoptosis was detected by flow cytometry in HGC-27 cells treated with oridonin for $24 \mathrm{~h}$ using an annexin V/PI staining method. The bar chart represents the percentage of cells undergoing apoptosis. (D) Oridonin blocks cell cycle at the $\mathrm{G}_{2} / \mathrm{M}$ phase. (E) Western blotting confirms downregulation of cyclin $\mathrm{B} 1$ and $\mathrm{CDK} 1$, and upregulation of $\mathrm{p} 53$ and $\mathrm{p} 21$. "P<0.05 and ${ }^{* * * *} \mathrm{P}<0.001$.

in HGC-27 cells by inducing apoptosis via cell cycle arrest. Furthermore, it was speculated that the proteins responsible for apoptosis such as cyclin B1, CDK1, p53 and p21 were involved in this process. Effectively, western blot analysis demonstrated downregulation of cyclin B1 and CDK1, and upregulation of p53 and p21 (Fig. 2E). 
A

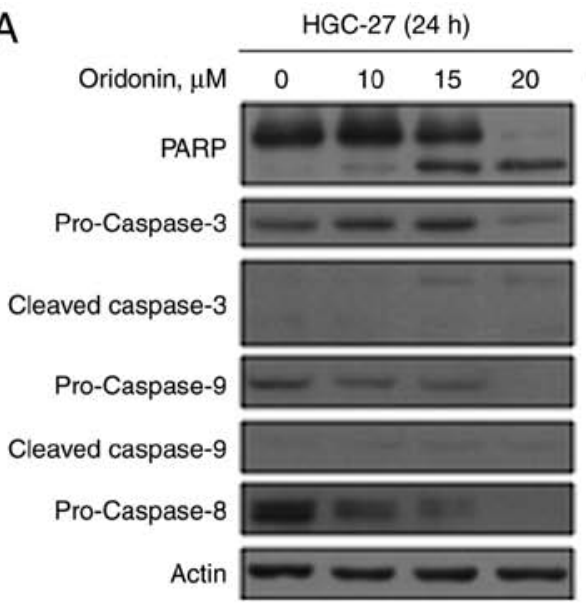

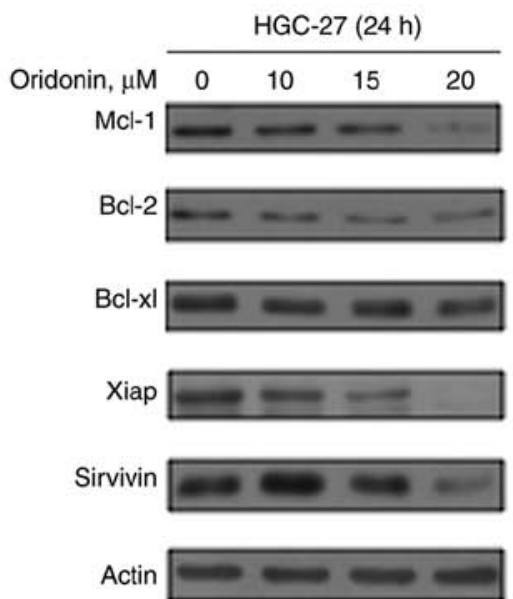

B
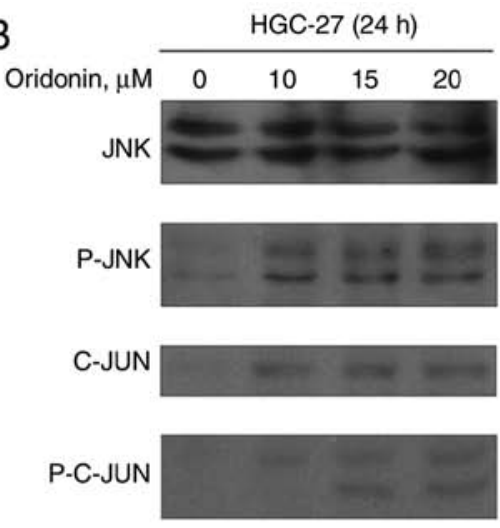

Actin

Figure 3. Oridonin induces caspase-dependent apoptosis in HGC-27 cells through the caspase-dependent p-JNK pathway (A) The effect of oridonin on caspase-associated proteins. (B) Western blot analysis confirming the activation of JNK and C-JUN. Following oridonin treatment, C-JUN activity was enhanced in a dose-dependent manner. p-, phosphorylated.
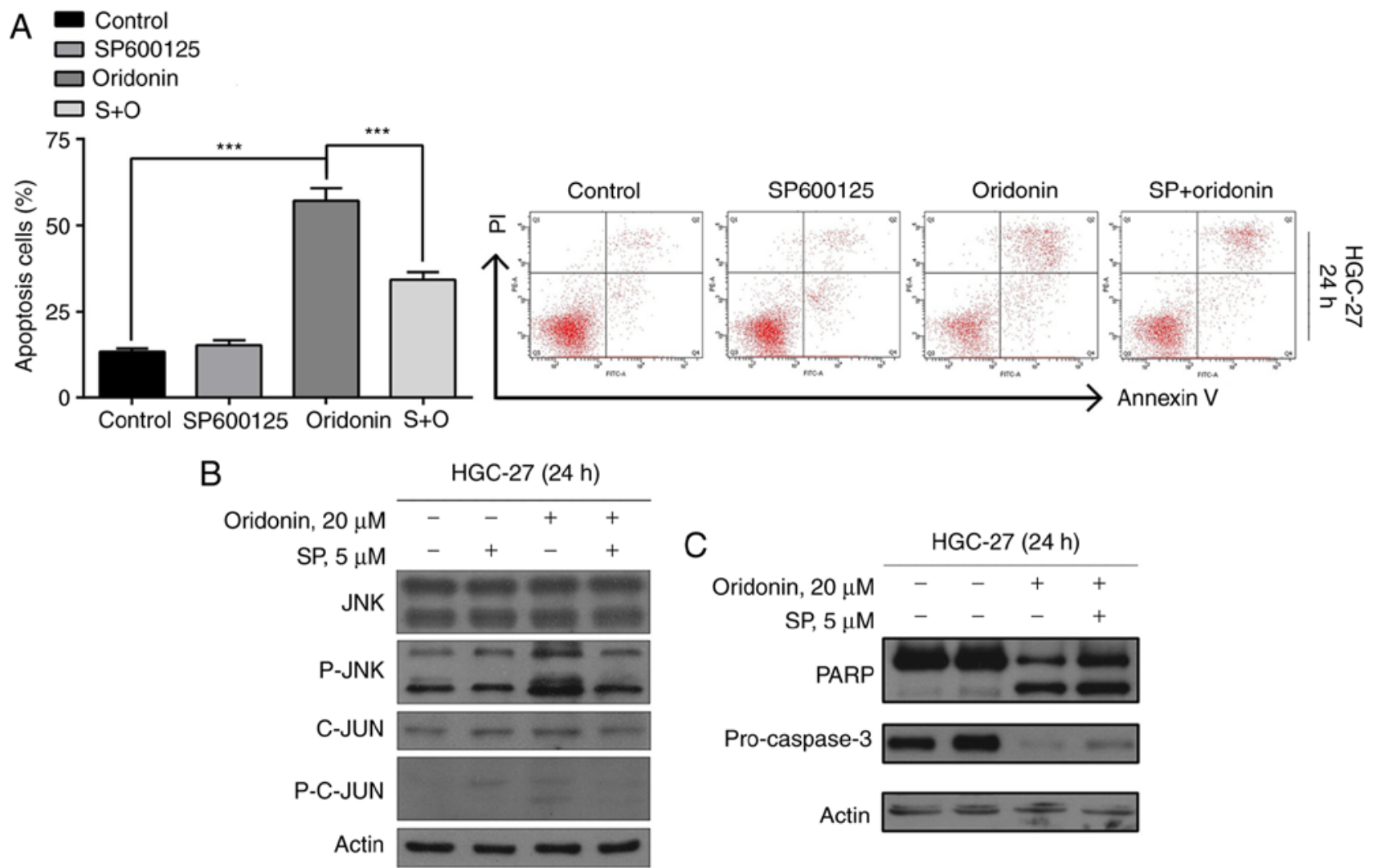

Actin

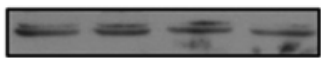

Figure 4. Effects of inhibiting p-JNK, C-JUN, p-C-JUN in oridonin-treated cells. (A) Administration of SP600125, a specific inhibitor of the JNK pathway, blocked the activation of JNK and lowered the rate of apoptosis induced by oridonin. Results from western blotting demonstrated that the oridonin-induced cleaved forms of (B) p-JNK, C-JUN, p-C-JUN, (C) PARP and the levels of precursor forms of caspase-3 were restored following addition of SP600125. ${ }^{* * * *} \mathrm{P}<0.001$. p-, phosphorylated.

Oridonin induces caspase-dependent apoptosis in HGC-27 cells through the JNK pathway. Several studies have reported that oridonin can induce apoptosis $(5,6)$. Following the treatment of HGC-27 cells with oridonin, dose-dependent downregulation of the precursor forms of caspase- $3,-8$, and -9 , and a dose-dependent upregulation of the cleaved forms of caspase- 3 , and -9 were observed (Fig. 3A). Furthermore, increased levels of PARP was also observed. Furthermore, downregulation of the anti-apoptotic proteins Mcl-1, Bcl-2, Bcl-xl, Xiap and survivin was also demonstrated (Fig. 3A).

Effects of inhibiting phosphorylated (p)-JNK, C-JUN,p-C-JUN in oridonin-treated cells. The effects of oridonin on the p-JNK pathway was subsequently evaluated. JNK and C-JUN were activated following oridonin treatment, and C-JUN activity was enhanced in a dose-dependent manner (Fig. 3B). 
Flow cytometric analysis demonstrated that the JNK pathway is involved in oridonin-induced apoptosis of GC cells. SP600125 was used as a general inhibitor of JNK. Effectively, SP600125 blocked the activation of JNK and lowered the rate of apoptosis induced by oridonin ( $\mathrm{P}<0.05$; Fig. 4A).

Furthermore, the results of the western blot analysis demonstrated that the oridonin-induced cleaved forms of p-JNK, C-JUN, p-C-JUN (Fig. 4B), PARP and the levels of precursor forms of caspase-3 (Fig. 4C) were restored upon the addition of SP600125. In summary, these observations suggest that oridonin induces apoptosis in HGC-27 cells via the p-JNK pathway.

\section{Discussion}

Oridonin is a commonly used drug in traditional Chinese medicine. Isolated from Rabdosiarubenscens, the medicinal drug was first recognized as having antitumor properties in 1967 (11). Since then, several studies have found the herb to exhibit good anticancer effects on a wide range of malignant cancer types such as colorectal cancer (12), esophageal cancer (13) and osteosarcoma (14). Previous findings have shown that oridonin triggers apoptosis in GC through the mitochondrial pathway (15). Herein, the findings of the present study demonstrated that oridonin can effectively arrest cells at the $\mathrm{G}_{2} / \mathrm{M}$ phase and induce apoptosis. Intrinsic apoptosis was demonstrated by decreased expression of the precursor levels of caspases-3, -8 , and -9 along with increased expression of cleaved forms of caspases-3, -8 and -9 . Extrinsic apoptosis activated the p-JNK/C-JUN pathway.

In the present study, decreased viability and proliferation of HGC-27 cells was demonstrated, following treatment with oridonin in a dose dependent manner. In eukaryotes, the cell cycle is controlled by cyclins and cyclin-dependent kinases (CDKs). Previous studies have found that cyclin B1 and CDK1 proteins actively partake in regulating the $\mathrm{G}_{2} / \mathrm{M}$ phase (16). The overexpression of cyclin $\mathrm{B} 1$ and CDK1 often results in uncontrolled cell growth. The treatment of HGC-27 cells with different concentrations of oridonin demonstrated the downregulation of cyclin B1 and CDK1, which led to a decreased proportion of cells at the $G_{2} / M$ phase. The findings of the present study are in accordance with previously published studies, where cell cycle arrest was also observed at the $\mathrm{G}_{2} / \mathrm{M}$ phase $(8,16)$.

Subsequently, the ability of oridonin to induce apoptosis in cells was evaluated. Apoptosis is an integral and highly preserved mode of cell death that is essential for normal development, host defense and inhibition of oncogenesis (6). Evading apoptosis is a distinct characteristic of all cancer cells; thus agents that can activate apoptosis in cancer cells are valuable in anticancer therapeutics. In the present study, apoptosis was evaluated by flow cytometry, by measuring the expression levels of anti-apoptotic and proapoptotic proteins. Flow cytometry initially inferred that oridonin caused apoptosis in HGC-27 cells. Programmed cell death was further confirmed upon decreased expression of the precursor levels of caspases-3, -8 , and -9 , increased expression of cleaved forms of caspases-3, -8 and -9 , as well as decreased expression of Mcl-1, Bcl-2 and Bcl-xl.

A significant increase in the pro-apoptotic Bcl-2 proteins results in the formation of pores in the outer mitochondrial membrane, which liberate apoptotic mitochondrial proteins to activate caspases and induce apoptosis. Caspases are activated during apoptosis in a self-amplifying cascade and play a potent role in programmed cell death. The increase in p53 triggered by increased expression of cleaved forms of caspases- 3 and -9 suggests the activation of the intrinsic apoptosis pathway by oridonin. Moreover, the downregulation of pro-caspase- 8 suggests that apoptosis was also triggered by the extrinsic pathway.

The findings of the present study show that oridonin triggers the p-JNK/C-JUN pathway, which is an upstream signal for caspase-associated apoptosis in HGC-27 cells. The association between the signaling pathways involved in oridonin-induced apoptosis should be further investigated. Further studies should focus on in vivo experiments to further investigate the anticancer mechanism of oridonin.

In conclusion, the present study indicates that oridonin can efficiently inhibit cell proliferation and induce caspase-dependent cell apoptosis by activating the $\mathrm{p}-\mathrm{JNK} / \mathrm{C}-\mathrm{JUN}$ pathway in HGC-27 cells. Moreover, these findings highlight the importance of targeting the JNK/C-JUN signaling pathway as an anticancer strategy. Thus, the potential of oridonin as an agent for chemotherapeutic therapy to treat GC was demonstrated.

\section{Acknowledgements}

Not applicable.

\section{Funding}

The present study was supported by the Science and Technology Program of Guangdong (grant no. 2017A010105004) and by Outstanding Young Reserve Talents Program of the Eighth Affiliated Hospital of Sun Yat-sen University (grant no. 2019006).

\section{Availability of data and materials}

The datasets used and/or analyzed during the current study are available from the corresponding author on reasonable request.

\section{Authors' contributions}

DR and RAG designed the experiments and wrote the manuscript. XW, QZ, and HC conducted the experiments, generated figures and contributed to the statistical analysis. XW first conceived the concept of this study, and ensured the accuracy and integrity of the manuscript. All authors read and approved the final version of the manuscript.

\section{Ethics approval and consent to participate}

Not applicable.

\section{Patient consent for publication}

Not applicable.

\section{Competing interests}

The authors declare that they have no competing interests. 


\section{References}

1. Siegel RL, Miller KD and Jemal A: Cancer Society, 2019. CA Cancer J Clin. 69: 7-34, 2019.

2. Van Cutsem E, Sagaert X, Topal B, Haustermans K and Prenen H: Gastric cancer. Lancet 388: 2654-2664, 2016.

3. Newman DJ and Cragg GM: Natural products as sources of new drugs from 1981 to 2014. J Nat Prod 79: 629-661, 2016.

4. Tan W, Lu J, Huang M, Li Y, Chen M, Wu G, Gong J, Zhong Z, $\mathrm{Xu} \mathrm{Z}$, Dang Y, et al: Anti-cancer natural products isolated from chinese medicinal herbs. Chin Med 6: 27, 2011.

5. Li Y, Wang Y, Wang S, Gao Y, Zhang X and Lu C: Oridonin phosphate-induced autophagy effectively enhances cell apoptosis of human breast cancer cells. Med Oncol 32: 365, 2015.

6. Bao R, Shu Y, Wu X, Weng H, Ding Q, Cao Y, Li M, Mu J, Wu W, Ding Q, et al: Oridonin induces apoptosis and cell cycle arrest of gallbladder cancer cells via the mitochondrial pathway. BMC Cancer 14: 217, 2014.

7. Cui Q, Tashiro S, Onodera S, Minami M and Ikejima T: Oridonin induced autophagy in human cervical carcinoma HeLa cells through Ras, JNK, and P38 regulation. J Pharmacol Sci 105: 317-325, 2007.

8. Sun KW, Ma YY, Guan TP, Xia YJ, Shao CM, Chen LG, Ren YJ, Yao HB, Yang Q and He XJ: Oridonin induces apoptosis in gastric cancer through Apaf-1, cytochrome c and caspase-3 signaling pathway. World J Gastroenterol 18: 7166-7174, 2012.

9. Wang H, Ye Y, Chui JH, Zhu GY, Li YW, Fong DW and Yu ZL: Oridonin induces $\mathrm{G} 2 / \mathrm{M}$ cell cycle arrest and apoptosis through MAPK and p53 signaling pathways in HepG2 cells. Oncol Rep 24: 647-651, 2010.

10. Burotto M, Chiou VL, Lee JM and Kohn EC: The MAPK pathway across different malignancies: A new perspective. Cancer 120: 3446-3456, 2014.
11. Fujita E, Nagao Y, Node M, Kaneko K, Nakazawa S and Kuroda H: Antitumor activity of the Isodon diterpenoids: Structural requirements for the activity. Experientia 32: 203-206, 1976.

12. Gao FH, Liu F, Wei W, Liu LB, Xu MH, Guo ZY, Li W, Jiang B and Wu YL: Oridonin induces apoptosis and senescence by increasing hydrogen peroxide and glutathione depletion in colorectal cancer cells. Int J Mol Med 29: 649-655, 2012.

13. Jiang JH, Pi J, Jin H and Cai JY: Oridonin-induced mitochondria-dependent apoptosis in esophageal cancer cells by inhibiting PI3K/AKT/mTOR and Ras/Raf pathways. J Cell Biochem 120: 3736-3746, 2019.

14. Wang XH, Zhang SF, Bao JT and Liu FY: Oridonin synergizes with Nutlin-3 in osteosarcoma cells by modulating the levels of multiple Bcl-2 family proteins. Tumour Biol 39: 1010428317701638, 2017.

15. Gao S, Tan H, Zhu N, Gao H, Lv C, Gang J and Ji Y: Oridonin induces apoptosis through the mitochondrial pathway in human gastric cancer SGC-7901 cells. Int J Oncol 48: 2453-2460, 2016.

16. Wang H, Zhu L, Feng X, Zhang H, Luo Q and Chen F: Oridonin induces $\mathrm{G} 2 / \mathrm{M}$ cell cycle arrest and apoptosis in human oral squamous cell carcinoma. Eur J Pharmacol 815: 282-289, 2017

This work is licensed under a Creative Commons Attribution-NonCommercial-NoDerivatives 4.0 International (CC BY-NC-ND 4.0) License. 